\title{
Real-time Acquisition System of Radar Echo Signal Based on PCI8001
} Gaofeng $\mathrm{CHENG}^{1, a}$, Ting SUN ${ }^{2, b}$, Yingming $\mathrm{LU}^{3, c}$, Shulai LIU ${ }^{4, d}$

1.4. Department of Control Engineering, Academy of Armored Forces Engineering, Beijing, China

2.Beijing Special Vehicle Academy, Beijing, 100072, China

3.Department of Training, Academy of Armored Forces Engineering, Beijing, China

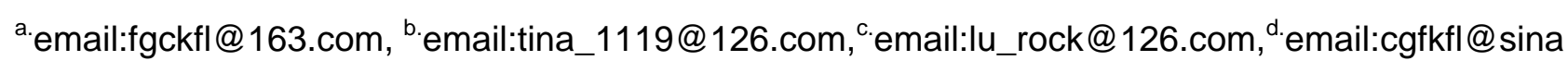
. $\mathrm{com}$

Keywords: Data acquisition; North error correction; Pseudo-colored fast algorithm

\begin{abstract}
In this paper, the design of real-time radar signal acquisition system based on domestic PCI8001 data acquisition card was introduced. By DMA, interrupting collected data was written to the industrial control computer with windows XP system. The technologies of multi-threaded processing, north error correction and pseudo-colored fast algorithm were used in software system. The acquisition of real-time radar video echo signal was realized by the system, display and storage. The system has been used in outside data collection of marine radar successfully.
\end{abstract}

\section{Introduction}

For a long time, acquisition of radar signal is based on imported data acquisition board. A high-speed,high-resolution data asquisition card PCI9820 of ADLINK is used in real-time acquisition system of radar echo signal [1] [2]. A high-speed system of radar signal collection and processing use $\mathrm{PCl} 2040$ of $\mathrm{TI}[3]$. These data acquisition board. are expensive.

This paper mainly describes a real-time radar echo signal acquisition system based on domestically PCI8001 data acquisition card. The system is capable of continuous acquisition, real-time display and real-time store the data from a certain type of imaging radar. System has sampling frequency of $40 \mathrm{MHz}$ with precision of 12 bit. Collected data are written to computer by DMA combining interrupt mode. After the software processing the radar data, an ordinary monitor can be used to real-time display in $\mathrm{P}$ or B way. The data recording, not only can be real-time manual storage, but also whole situation continuous and automatic storage.

\section{System Structure}

The system is based on industrial control computer with windows XP OS, acquires radar video echo signals through the PCI8001 acquisition card. PCI8001 controls the radar video echo signal acquisition according to the trigger signal from radar system and the antenna's north signal. Acquired data are transferred to computer's memory via computer's PCI interface for data processing and display. Finally system stores the data collected on a SATA-II disk in real time. System structure shown in Figure [1].

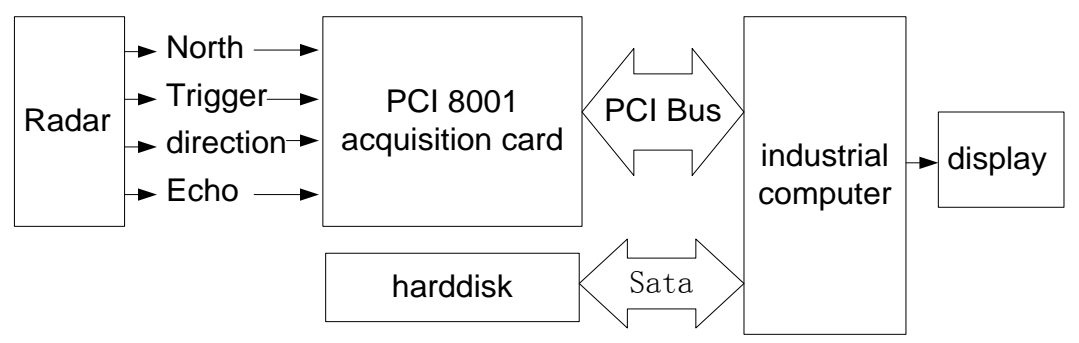

Figure 1. Acquisition System Structure 


\section{Data acquisition card PCI8001 Features}

\section{A. Board-related Features}

PCI8001 card is a high-performance data acquisition card based on 32-bit PCI bus architecture. It has the sample frequency of $80 \mathrm{M} / \mathrm{n}$ (where $\mathrm{n}$ is any even number between 2 and 254), and a total of four analog input channels, each has 512K word cache. PCI8001 supports multiple trigger modes: software directly trigger, external digital signal rising edge trigger, external digital signal falling edge trigger, external analog signal trigger. It can be used flexible.

\section{B. Real-time Driven}

A normal DLL library direct calls the dynamic simply, putting the hardware control and data transmission code in the DLL. The code has the user program execution priority level, and should be called by the system-level task scheduler regularly and continuously, which is likely to cause data lose. To solve these problems, in the NT environment, the card put the hardware control and data transmission code into the NT kernel in the form of micro-kernel code utilizing the microkernel technology. Becoming a part of the NT operating system makes the program works on high priority and speed, which greatly improves the quality of the data collection and transmission.

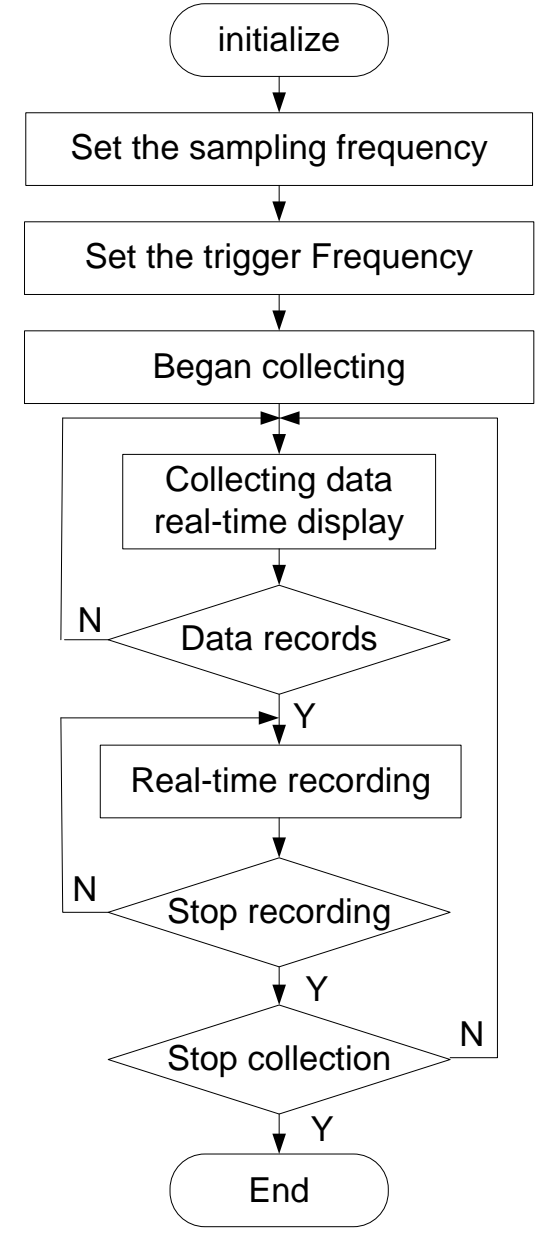

Figure 2. System workflow

\section{PCI8001 real-time acquisition program}

\section{A. Program design and flow chart}

It is required that this system is able to display acquired radar video echo signal on an ordinary monitor. At the same time during the display, the acquisition trigger frequency and acquisition frequency can be set and the acquired data is stored on disk in real time. While working in the acquisition state, the acquisition card acquire and transfer the 360-degree, full distance radar video 
echo signal to the computer's memory. It is displayed in P or B way in full screen on the monitor.

While recording the data, both the real-time manual storage of the focused situation and automatical, continuous whole situation storage can be used。The system flow chart is shown in Figure 2.

\section{B. Multithreaded program design}

A large amount of radar data acquisition and display is easy to produce leakage and other issues because of the low priority of common programs in windows XP system. The process priority HIGH_PRIORITY_CLASS should be set using the SetPriorityClass() function. In order to deal with the contradiction between the requirements of real-time data acquisition and the time assuming issue of the display and data processing, a separate data acquisition thread is created while data processing and display control are realized in the main process. A data acquisition and window drawing object synchronization event hExitEvent = PCI8001_CreateSystemEvent () is created. Waiting acquisition thread WaitForSingleObject (hExitEvent, 100) is used when closing the the acquisition thread to guarantee the thread safely exits.

Radar signal acquisition thread is only responsible for collecting radar data. PCI8001 radar data acquisition thread flow chart is shown in Figure 3. Acquisition The north, the orientation, and radar echo signals are acquired simultaneously and are real-time passed to the main process for data processing and display.

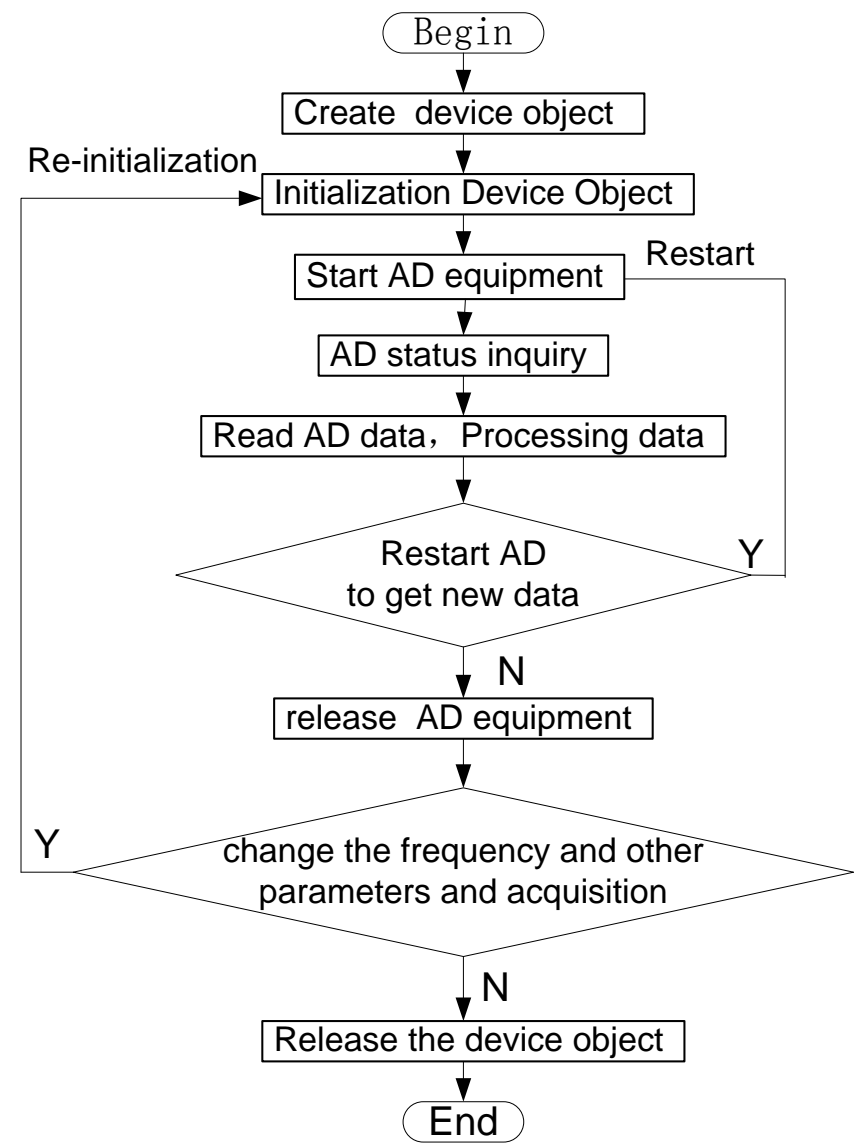

Figure 3. $\quad$ Radar data acquisition thread flow chart

\section{North judgment and correction}

True north radar pulse is the reference of the radar data processing, however it is found that in practice the north radar pulse is instable and it is difficult to determine the exact time of the north because of the large rising slope of the north pulse. A joint algorithm using mean filtering and signal error correction of the north signal is adopted. The north pulse signal can be well corrected. 


\section{A fast algorithm for pseudo-color}

In a traditional color radar image algorithm, the radar echo amplitude is directly converted to a color chart, however it is found in a marine radar verification that the displayed radar image contrast is low and it is difficult to see the target clearly. The experimental radar echo is converted into decibel, divided into 24 aliquots and then converted to a pre-defined color. The image is clear using this method. But for the massive radar image data, especially for large computation of multiplication, division and logarithm, impacting the real-time image display. In order to deal with this, the second look-up table method using the data tables computed during design is adopted. The pseudo-color calculation is accomplished using only two simple reference counting. The algorithm is fast and can meet the needs of imaging radar image display.

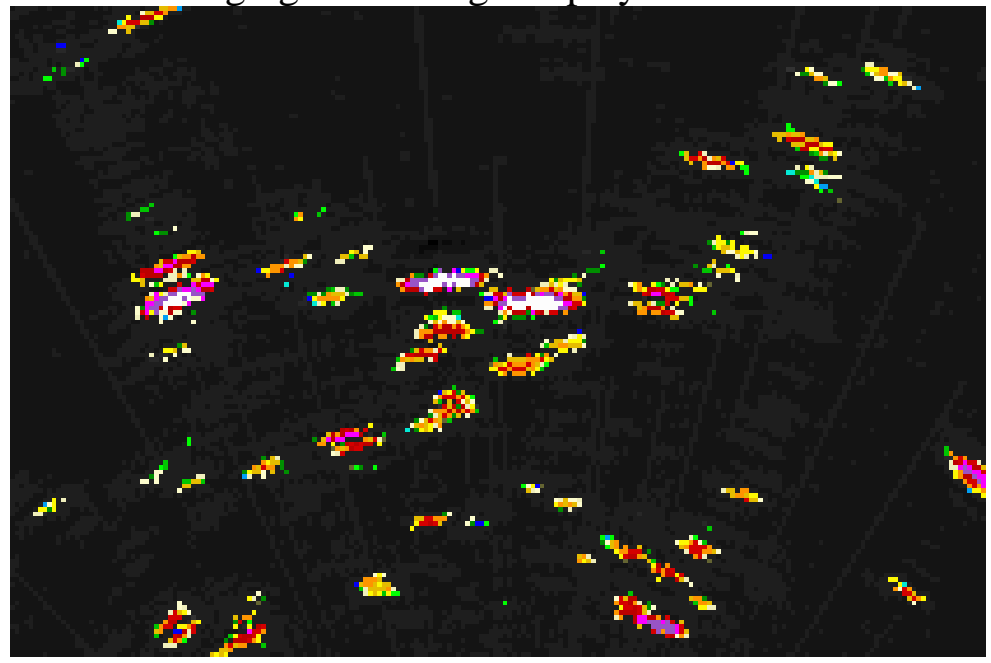

Figure 4. Radar pseudo-color image

\section{Effect}

The real-time acquisition system of radar echo signal based on PCI8001 presented has been applied in a marine radar field. As shown in Figure 5, the results of the practical application shows that the system meets the need of real-time radar video echo signal acquisition, display and storage.

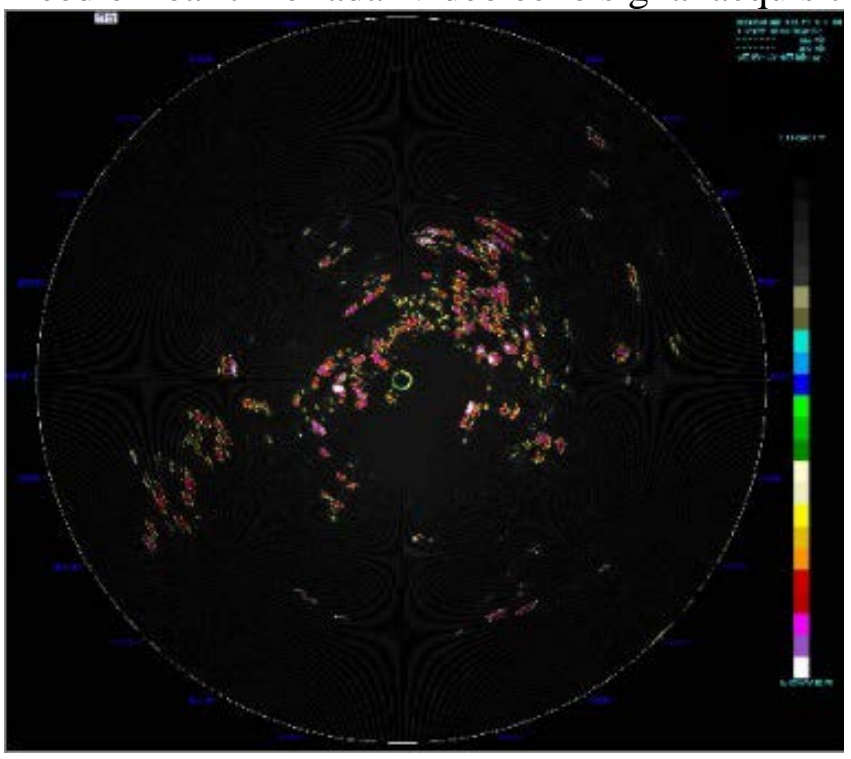

Figure 5. Radar display in P way

\section{Summary}

All-digital display is the inevitable trend of the development of radar terminal displays. The acquisition system of radar echo signal Based on PCI8001 can realize real-time storage and 
All-digital display.

\section{References}

[1] Li Fu-qiang, Wang Hong, Zeng Qing-shan ,Lu Yi ,Real-time Acquisition System of Radar Echo Signal Based on PCI9820 [J], Microcomputer Information, 2005(6) 69-71.

[2] Wan Hong,Li Shen-tang,Wang Zhi-gang, Application of High-speed Acquisition Card to Sampling of Radar Signal[J], Moden Radar, 2005 27(12) 84-90.

[3] Gu Zhi-feng,Dai Chun-ni,Xiao Li-min,High-Speed System of Radar Signal Collection \& Processing Based on PCI2040 and TMS320C6201[J],Journal of Guangxi University For Nationalities,2005 11(3) 77-81.

[4] YU Hui-ying,Lu Min,YONG Shao-wei, Design and Realization of PPI Signal Raster Scan Radar Display System[J], Journal of National University of Defense Technology, 2007 29(1) 65-68.

[5] Brakat M,Himonas S F,Varshney Pk,CFAR detection for Multiple Target Situations.IEEE Proc-F,1989 136(5) 193-209.

[6] He Qi-yuan,Zhang Zun-quan,Raster Scan Displaying System Based on Dirert X and PCI Bus[J], Moden Radar, 2003 25(2) 54-56. 